\title{
Comparison Accuracy in mSIRS, NEWS and qSOFA Score to Triage Sepsis Patients in The Emergency Department at a Hospital in Thailand
}

Jiratti Jaruwatthanasunthon, M.D., Panita Worapratya, M.D., Thammapad Piyasuwankul, M.D.

Department of Emergency Medicine, Faculty of Medicine, Prince of Songkla University, Songkhla 90110, Thailand.

Received 12 December 2020 • Revised 2 October 2021 • Accepted 8 November 2021 • Published online 11 January 2022

\begin{abstract}
:
Objective: We aimed to apply the modified systemic inflammatory response syndrome (mSIRS), the quick sequential organ failure assessment score (qSOFA), and National Early Warning Score (NEWS) to triage suspected sepsis patients. Therefore, knowing the predictive performance of each scoring system, using given cut-points for triaging patients with suspected sepsis, could help predict the progression of sepsis.

Material and Methods: This study is a single-center retrospective chart review. The study enrolled patients older than 18 years with suspected sepsis patient at the time they presented at the triage zone. The primary outcome was to determine which scoring system were the most accurate to triage sepsis patients. The secondary outcomes were predictions of mortality related to the scoring.

Results: Considering the outcome to be represented by a SOFA score of $\geq 2$, the area under the curve of the receiver operating characteristic curves for the entire range of mSIRS, qSOFA and NEWS were 0.494, 0.669 and 0.751 , respectively. Using a cut point for qSOFA of $\geq 2$ provided a low sensitivity of $36.2 \%$ and high specificity of $93.0 \%$; whereas, using a cut point for NEWS of $>4$ provided a high sensitivity of $89.0 \%$ and low specificity of $33.0 \%$.

Conclusion: In summary, qSOFA is the most accurate scoring system for diagnosis sepsis which was consistent with previous study. However, qSOFA had the lowest sensitivity, so is not appropriate in a triage situation. Therefore, we decided to use NEWS as the triage tool because of its better sensitivity and acceptable specificity as we need to triage almost all possible cases.
\end{abstract}

Keywords: Emergency Department, NEWS score, SOFA score, Triage sepsis

Part of this work was poster presented at the $18^{\text {th }}$ International Conference on Emergency Medicine [ICEM] $12^{\text {th }}-15^{\text {th }}$, June, 2019

Contact: Jiratti Jaruwatthanasunthon, M.D.

Department of Emergency Medicine, Songklanagarind Hospital, Faculty of Medicine,

Prince of Songkla University, Songkhla 90110, Thailand.

E-mail: jiratti.eppsu@gmail.com

(c) 2021 JHSMR. Hosting by Prince of Songkla University. All rights reserved.

This is an open access article under the CC BY-NC-ND license

(http://www.jhsmr.org/index.php/jhsmr/about/editorialPolicies\#openAccessPolicy).
J Health Sci Med Res 2022;40(5):487-495 doi: 10.31584 /jhsmr.2021858 www.jhsmr.org 


\section{Introduction}

According to the Third International Consensus Definition for Sepsis and Septic Shock (Sepsis-3), sepsis is defined as a "life-threatening organ dysfunction caused by a dysregulated host response to infection" and organ dysfunction is defined as "an increase in the Sequential (Sepsis-related) Organ Failure Assessment (SOFA) score of 2 points or more." which is a gold standard nowadays. ${ }^{1}$ However, in reality, triaging patients with suspected sepsis is difficult because sepsis is internal organ damage that cannot be seen with the naked eye. We need many laboratory investigations to diagnose sepsis according to Sepsis-3, such as arterial blood gas results, complete blood count, and electrolyte and liver function test results. Therefore, we cannot triage patients with suspected sepsis at the triage zone with the Sepsis-3 definition.

A parsimonious clinical model developed from Sepsis-3 with multivariable logistic regression indicated that any 2 of 3 clinical variables (i.e. Glasgow Coma Scale (GCS) score $\leq 13$, systolic blood pressure $\leq 100 \mathrm{~mm} \mathrm{Hg}$, and respiratory rate $\geq 22 / \mathrm{min}$ ) offered predictive validity similar to that of the full SOFA score outside the intensive care unit (ICU). ${ }^{1,2}$

But outside the ICU sepsis group, only 58.3\% met the quick SOFA (qSOFA) criteria $^{3}$ and a recent systematic review and data from a meta-analysis showed that the qSOFA criteria used as a screening tool in the emergency room had poor sensitivity $(0.51) .{ }^{4}$ However, recent data seemed to indicate that the sensitivity of the national early warning score (NEWS) was superior to the modified early warning score and the qSOFA score. The sensitivity of NEWS $\geq 5$ is similar to the systemic inflammatory response syndrome criteria of $\geq 2$ and higher than qSOFA $\geq 2$. Furthermore, NEWS had a similar Area Under the Receiver Operating Characteristics (AUROC) to qSOFA. ${ }^{5}$

We found few studies on triage score comparisons that were accurate and easy to apply in patients with suspected sepsis, especially in the emergency triage zone. Moreover, no such study has been reported in Asia including Thailand. Therefore, we performed a retrospective comparison between the SIRS, NEWS, and qSOFA scores to triage septic patients in the Emergency Department (ED) at Songklanagarind Hospital in southern Thailand.

Sepsis is an important public health problem in many countries. The incidence of sepsis varies in many studies. The incidence of sepsis is 77 per 100,000 in Australia and New Zealand ${ }^{6}$ and 240 per 100,000 in the United States of America (USA). ${ }^{7}$ The running costs of sepsis were 20 billion US dollars or equal to $5.2 \%$ of all running costs of hospitals in the USA in $2013^{1}$ and has also become the 10th leading cause of death in the USA. ${ }^{8}$

Rapid recognition, early resuscitation, early antibiotics, and eradication of the source of infection are the key components in the quality of sepsis care and have been proven significant in decreasing mortality ${ }^{9-11}$ but the definition of Sepsis-3 delays the management of sepsis because it is based on laboratory data. Therefore, this study aimed to find tools to help in the early detection of sepsis.

We aimed to apply the modified systemic inflammatory response syndrome (mSIRS), qSOFA, and NEWS scores as guidelines to triage patients with suspected sepsis. Therefore, knowing the predictive performance of each scoring system, using given cut-points for triaging patients with suspected sepsis in our setting, could help predict the progression of sepsis. This would lead to a treatment guideline which is rapid, correct, and appropriate for patient care.

\section{Material and Methods}

This study is a single-center retrospective chart review. The study was conducted in the ED of Songklanagarind Hospital which is an academic, tertiary care hospital on the campus of Prince of Songkla University in southern Thailand. The hospital is also the referral center 
in southern Thailand for critically ill and complicated patients. The ED treats approximately 25,000 patients per year. The institutional review board of the Faculty of Medicine, Prince of Songkla University approved this study (EC. 60-41620-4).

We reviewed the charts of patients who visited the ED from January 2017 to May 2018. The study enrolled patients older than 18 years, with suspected sepsis from vital signs, history taking, physical examination and investigation at the time they presented at the triage zone, or were detected soon after arrival in the ED. We excluded trauma patients, patients without at least two bottles for hemoculture, and patients whose final diagnosis was not bacterial infection. Also excluded were those patients whose vital signs or triage scores were not completely recorded in the chart.

The initial vital signs and clinical presentation data at the triage zone were recorded by a triage nurse. The mSIRS, qSOFA, and NEWS scores were calculated by two researchers. Other data reviewed were the arrival time, time in the emergency room (ER), time to receive antibiotics, time to receive fluid resuscitation, diagnosis, hemoculture results, organ-specific morbidity, and discharge status. The scores were registered in the electronic medical record. The data were cross-checked before encoding into the database by a research assistant.

The charts were reviewed until the patient was discharged from the hospital after the same ED visit. The primary outcome was to determine which scoring system (mSIRS, qSOFA and NEWS) were the most accurate to triage sepsis patients compared with SOFA score, which is currently the gold standard. The secondary outcomes were predictions of mortality related to the scoring.

Initial vital signs were defined as body temperature, pulse rate, respiratory rate, blood pressure, oxygen saturation, and GCS score at the initial presentation in the triage zone. The mSIRS score cut-point was identified as any 2 of 3 clinical variables [i.e. tachycardia [heart rate $>90$ beats/min], tachypnea (respiratory rate $>20$ breaths/min), and fever or hypothermia (temperature $>38^{\circ} \mathrm{C}$ or $<36^{\circ} \mathrm{C}$ )] We excluded white blood cell counts because they were not available in the triage area. The qSOFA score cut-point was identified as any 2 of 3 clinical variables (i.e. GCS score of $\leq 13$, systolic blood pressure of $100 \mathrm{~mm} \mathrm{Hg}$ or less, and respiratory rate $\geq 22 / \mathrm{min})$. The NEWS cut-point was identified as a summation of NEWS $>4$ ( 5 or more), which was the medium score according to The United Kingdom, Royal College of Physicians 2012. Immunocompromised patients were defined as immunodeficiency from any cause or the use of immunosuppressive drugs or both. Central venous access was defined as permanent catheter insertion at the jugular vein, subclavian vein or femoral vein. Endstage renal disease was defined as patients undergoing dialysis via permanent catheter, arteriovenous fistula or peritoneal dialysis. Diabetes mellitus included insulindependent and non-insulin-dependent types. Cancer was defined as a neoplasm of any type. Chemotherapy was defined as last chemotherapy within the previous 6 months. Immunosuppressive drug use was defined as prednisolone $>15 \mathrm{mg} /$ day or on oral chemotherapy. Acquired immunodeficiency syndrome was defined as cluster of differentiation 4 (CD4) cell count $<200$ cells $/ \mathrm{mm}^{3}$.

All data were entered into Epidata software version 3.1 and the statistical analysis used $R$ software version 3.2.2 and STATA software version 14.1. The continuous variables were analyzed and reported as mean and median while categorical variables were reported as percentage. The numbers were rounded up or down to the nearest 0.5 integer.

Receiver operating characteristics (ROC) curves were constructed for each of the scoring scales, and areas under the curve (AUC) were compared. Cut-point values 
of each scoring scale, as reported in the literature, were initially applied to provide a binary test, sensitivity, specificity, likelihood ratio positive and negative estimation. Predictive values for the outcome of a SOFA score of $>2$ of a positive or negative test result, as applied to the current dataset, were calculated for each scoring system.

Mortality associated with positive and negative test results of each scoring system, with initial and subsequent additional cut-points, was estimated and also reported as likelihood ratios and risk ratios, and for the current dataset as predictive values. A $p$-value $<0.050$ was considered statistically significant.

\section{Results}

A total of 502 patients were screened for the study and 38 patients were excluded because the final diagnosis was viral infection (e.g., influenza, dengue fever, and common cold). We also excluded 46 patients who did not have at least two bottles for hemoculture. Finally, 418 patients were enrolled into the study. The flow chart of patient enrollment is shown in Figure 1.
Four hundred and eighteen subjects were enrolled into this study. The median age was 66 years old. Men comprised $54.8 \%$ of the 418 patients while $50.7 \%$ were immunocompromised hosts. Advanced cancer or previous chemotherapy or both within the previous 6 weeks comprised $23.7 \%$ of the immunocompromised hosts. The median mSIRS was 3, median qSOFA was 1 , median NEWS was 6 , and the median SOFA was 2 . The most common organ-specific infection was pulmonary (27.3\%) followed by urinary tract (25.8\%). The mortality rate was $11.2 \%$ (Table 1 ).

From the 418 patients analyzed, $218(52.2 \%)$ had a SOFA score $\geq 2$, and were considered as actually having sepsis.

Considering the outcome to be represented by a SOFA score of $\geq 2$, the AUC (95\% confidence interval) of the ROC curves for the entire range of mSIRS, qSOFA and NEWS were 0.494 (0.445, 0.543$), 0.669$ (0.630, 0.709) and $0.751(0.705,0.797)$, respectively. This revealed the mSIRS scales lack of discriminating ability (Figure 2).

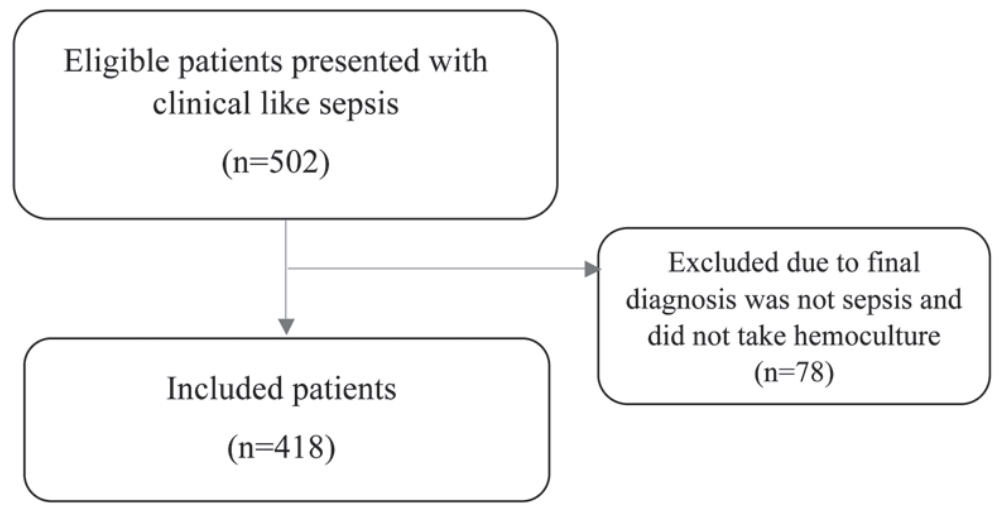

Figure 1 Patient enrollment flow chart 
Table 1 Demographic and baseline characteristics

\begin{tabular}{ll}
\hline Characteristics & $\begin{array}{l}\text { Entire study } \\
(\mathbf{n}=418)\end{array}$ \\
\hline Age, year, mean (S.D.) & $62.3(19.3)$ \\
Male sex, $\mathrm{n}(\%)$ & $229(54.8)$ \\
Immunocompromised, $\mathrm{n}(\%)$ & $212(50.7)$ \\
Central venous catheter & $2(0.9)$ \\
End stage renal disease & $23(10.8)$ \\
HCC/cirrhosis child C & $7(3.3)$ \\
Diabetes & $70(33.0)$ \\
Cancer or last chemotherapy <6 week & $99(46.7)$ \\
On immunosuppressive & $16(7.5)$ \\
Human immunodeficiency virus & $5(2.4)$ \\
Splenectomy & $1(0.5)$ \\
Emergency severity index level, $n(\%)$ & \\
1 & $20(4.8)$ \\
2 & $255(61)$ \\
3 & $136(32.5)$ \\
4 & $7(1.7)$ \\
Triage score, median & \\
SIRS score & 3 \\
mSIRS score & 3 \\
qSOFA & 1 \\
SOFA & 2 \\
NEWS & 6 \\
\hline
\end{tabular}

Table 1 (Continued)

\begin{tabular}{ll}
\hline Characteristics & $\begin{array}{l}\text { Entire study } \\
(\mathbf{n = 4 1 8})\end{array}$ \\
\hline Source of infection, $\mathrm{n}(\%)$ & \\
$\quad$ Primary bacteremia & $42(10.0)$ \\
Non identify & $28(6.7)$ \\
Lungs & $114(27.3)$ \\
Urinary tract & $108(25.8)$ \\
Skin & $35(8.4)$ \\
Gastrointestinal & $78(18.7)$ \\
Central nervous system & $3(0.7)$ \\
Other & $21(5.0)$ \\
Hemoculture positive, $\mathrm{n}(\%)$ & $82(19.6)$ \\
Results, $\mathrm{n}$ (\%) & \\
Discharge by improved & $313(74.9)$ \\
Death & $47(11.2)$ \\
Refer & $58(13.9)$ \\
\hline
\end{tabular}

S.D.=standard deviation, HCC=Hepatocellular carcinoma, mSIRS $=$ modified systemic inflammatory response syndrome, qSOFA=quick sequential organ failure assessment, SOFA=sequential organ failure assessment, NEWS=National Early Warning Score

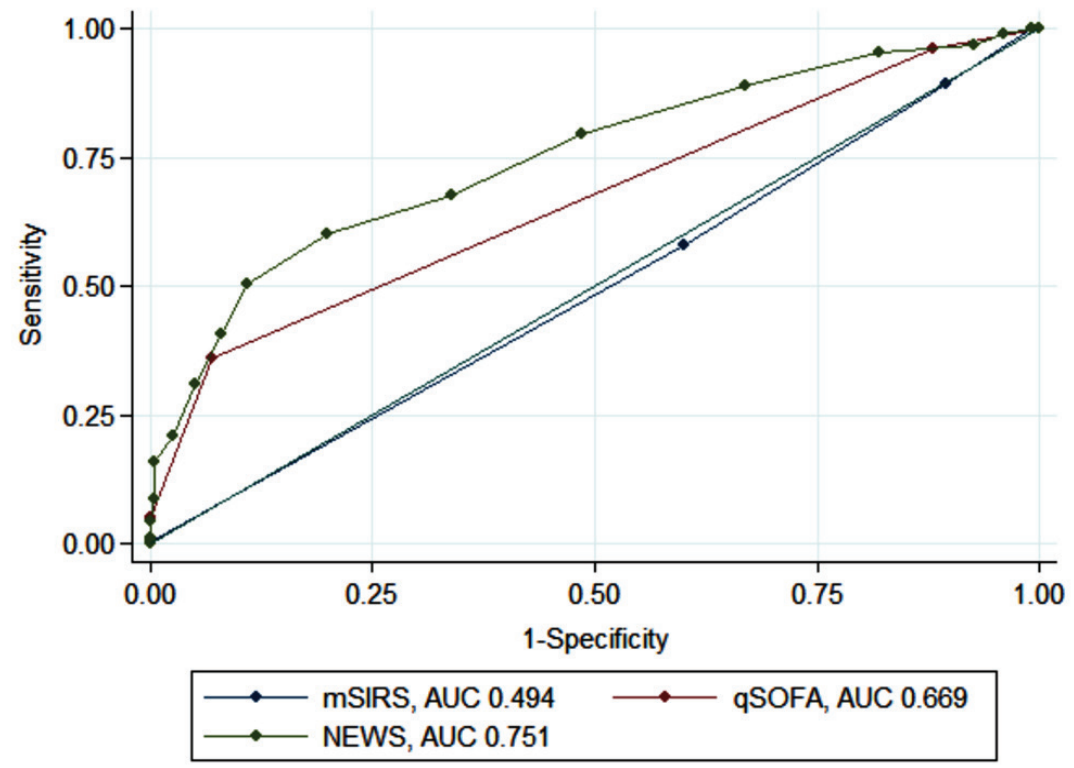

Figure 2 ROC curves of basic scores of mSIRS, qSOFA and NEWS for prediction of sepsis, defined as SOFA $\geq 2$ 
Using a cut point for qSOFA of $\geq 2$ provided a low sensitivity of $36.2 \%$ and high specificity of $93.0 \%$; whereas, using a cut point for NEWS of $>4$ provided a high sensitivity of $89.0 \%$ and low specificity of $33.0 \%$. Values of likelihood ratio both positive and negative, were 5.18 and 0.69 for qSOFA and 1.33 and 0.33 for NEWS, respectively (Table 2).

In addition, we also estimated the predictive performance of $m S I R S \geq 2$, qSOFA $\geq 2$, NEWS $>4$, as well as SOFA $\geq 2$ with mortality. A SOFA $\geq 2$ had the highest risk ratio $(R R)$ of 6.27 (2.72, 14.49; p-value $<0.001)$, followed by NEWS $>4$, with an RR of $4.02(1.27,12.66$; $p-$ value $=$
$0.007)$; and qSOFA $\geq 2$ with RR of 3.97 (2.35, 6.71; p-value $<0.001)$. Meanwhile, mSIRS had the lowest RR of $1.26(0.48$, 3.36), which was not statistically significant (Table 3).

With the additional cut points for NEWS at $>7$ and $>8$, the percentages of mortality in the three levels using the additional cut-point of $>7$ were $3.3 \%, 4.5 \%$ and $21.6 \%$, with, respectively, relative risks of $1,1.34$ and 6.49 ; and likelihood ratios of $0.03,0.04$ and 2.18 (Table 4). Using the additional cut-point of $>8$, the percentages of mortality were $3.3 \%, 4.6 \%$ and $26.5 \%$, relative risks were $1,1.38$ and 7.95 , and likelihood ratios were $0.27,0.38$ and 2.85 , respectively (Table 5 ).

Table 2 Scores of mSIRS, qSOFA and NEWS to predict sepsis defined by SOFA score $\geq 2$

\begin{tabular}{|c|c|c|c|c|c|c|c|}
\hline \multirow{2}{*}{ Scale } & \multirow{2}{*}{ Level } & \multicolumn{2}{|c|}{ SOFA } & \multirow{2}{*}{ LR } & \multirow{2}{*}{$\begin{array}{l}\text { Sepsis } \\
\text { proportion }\end{array}$} & \multirow{2}{*}{ Sensitivity } & \multirow{2}{*}{ Specificity } \\
\hline & & $\geq 2$ & $<2$ & & & & \\
\hline \multirow[t]{2}{*}{ mSIRS } & $\geq 2$ & 195 & 179 & 1.00 & 0.52 & 0.89 & 0.11 \\
\hline & $<2$ & 23 & 21 & 1.00 & 0.48 & & \\
\hline \multirow[t]{2}{*}{ qSOFA } & $\geq 2$ & 79 & 14 & 5.18 & 0.85 & 0.36 & 0.93 \\
\hline & $<2$ & 139 & 186 & 0.69 & 0.43 & & \\
\hline \multirow[t]{2}{*}{ NEWS } & $>4$ & 194 & 134 & 1.33 & 0.59 & 0.89 & 0.33 \\
\hline & $\leq 4$ & 24 & 66 & 0.33 & 0.27 & & \\
\hline
\end{tabular}

LR=Likelihood ratio, mSIRS=modified systemic inflammatory response syndrome, qSOFA=quick sequential organ failure assessment, NEWS=National Early Warning Score

Table 3 Scores of mSIRS, qSOFA, NEWS and SOFA, each with one cut-point to predict mortality

\begin{tabular}{lllllllll}
\hline Scale & Level & Dead & Survive & LR & $\begin{array}{l}\text { Mortality } \\
\text { proportion }\end{array}$ & RR & 95\% Cl & p-value \\
\hline mSIRS & $\geq 2$ & 43 & 331 & 1.02 & 0.25 & 1.26 & $0.48,3.36$ & 0.630 \\
& $<2$ & 4 & 40 & 0.79 & 0.09 & & & \\
qSOFA & $\geq 2$ & 25 & 68 & 2.90 & 0.27 & 3.97 & $2.35,6.71$ & $<0.001$ \\
& $<2$ & 22 & 303 & 0.57 & 0.07 & & & \\
NEWS & $>4$ & 44 & 284 & 1.22 & 0.13 & 4.02 & $1.27,12.66$ & 0.007 \\
& $<4$ & 3 & 87 & 0.27 & 0.03 & & & \\
SOFA & $\geq 2$ & 41 & 177 & 34.08 & 0.19 & 6.27 & $2.72,14.49$ & $<0.001$ \\
& $<2$ & 6 & 194 & 0.24 & 0.03 & & & \\
\hline
\end{tabular}

$\mathrm{LR}=$ Likelihood ratio, $\mathrm{RR}=$ risk ratio, $\mathrm{Cl}=$ confidence interval, $\mathrm{mSIRS}=$ modified systemic inflammatory response syndrome, qSOFA=quick sequential organ failure assessment, NEWS=National Early Warning Score, SOFA=sequential organ failure assessment 
Table 4 NEWS with two cut-points ( $>4$ and $>7$ ) to predict mortality

\begin{tabular}{lllllllll}
\hline Scale & Level & Dead & Survive & LR & $\begin{array}{l}\text { Mortality } \\
\text { proportion }\end{array}$ & RR & 95\% Cl & p-value \\
\hline NEWS & $>7$ & 37 & 134 & 2.18 & 0.22 & 6.49 & $2.06,20.47$ & $<0.001$ \\
& $>4, \leq 7$ & 7 & 150 & 0.37 & 0.04 & 1.34 & $0.35,5.04$ & \\
& $\leq 4$ & 3 & 87 & 0.27 & 0.03 & 1.00 & & \\
\hline
\end{tabular}

$\mathrm{LR}=\mathrm{Likelihood}$ ratio, $\mathrm{RR}=$ risk ratio, $\mathrm{Cl}=$ confidence interval, NEWS=National Early Warning Score

Table 5 NEWS with two cut-points $(>4$ and $>8$ ) to predict mortality

\begin{tabular}{lllllllll}
\hline Scale & Level & Dead & Survive & LR & $\begin{array}{l}\text { Mortality } \\
\text { proportion }\end{array}$ & RR & $95 \%$ Cl & p-value \\
\hline NEWS & $>8$ & 35 & 97 & 2.85 & 0.26 & 7.95 & $2.52,25.08$ & $<0.001$ \\
& $>4, \leq 8$ & 9 & 187 & 0.38 & 0.05 & 1.38 & $0.38,4.97$ & \\
& $\leq 4$ & 3 & 87 & 0.27 & 0.03 & 1.00 & & \\
\hline
\end{tabular}

LR=Likelihood ratio, RR=risk ratio, $\mathrm{Cl}=$ confidence interval, NEWS=National Early Warning Score

\section{Discussion}

This present study showed that the AUROC of the original scores of both NEWS (0.751) and qSOFA (0.669) were quite high, while that of mSIRS (0.494) showed that mSIRS totally lacked any discriminating ability for sepsis. qSOFA $\geq 2$ had high AUROC to predict a septic patient with Sepsis-3 criteria, which is consistent with previous studies. $^{2,12}$ and also had the highest likelihood ratio+ (LR+) which meant that a patient with qSOFA $\geq 2$ had a high probability for a diagnosis of sepsis. Even though qSOFA $\geq 2$ had the highest LR+ and high AUROC, it also had the lowest sensitivity which implied that two-thirds of septic patients outside the intensive care unit could be missed, which is consistent with previous studies. ${ }^{13-15}$

mSIRS seemed to be useless for sepsis triage. It predicted mortality because its overestimated sepsis and the specificity was very low. Furthermore, the likelihood ratio showed no statistical change which was consistent with previous studies. ${ }^{14,15}$
NEWS cut at $>4$ had high sensitivity (0.89), but low specificity (0.33) and a LR+ of 1.33 , while mSIRS cut at $\geq 2$ had a higher specificity than mSIRS; however, it was still low. Nevertheless, in triage septic patients we need to triage almost every possible case. Therefore, we decided to use NEWS as the triage tool because it had a sensitivity as high as mSIRS although it had low specificity which was better than qSOFA with a low sensitivity. However, a high specificity is not appropriate in a triage situation.

The NEWS cut-point also indicated whether the patient should stay in the emergency zone or urgency zone as predicted by mortality. In predicting mortality, we found that SOFA $\geq 2$ had the highest RR (6.27) followed by NEWS $>4$ (4.02) and qSOFA $\geq 2$ (3.97) (Table 3). This indicated that SOFA $\geq 2$ was the best score to predict mortality but the SOFA score cannot be evaluated by the naked eye during transfer to the ER since the SOFA score is determined from internal organ damage. 
Also, NEWS could predict mortality; however, due to the high sensitivity, NEWS $>4$ may give many false positive results. Transferring all suspected septic patients with a NEWS $>4$ into the emergency zone may cause problems related to limited resources (e.g., equipment for monitoring, medical personnel per patient while monitoring, and close monitoring in patients with a final diagnosis of SOFA $<2$ after investigations).

We also explored different cut-points of NEWS for predicting mortality (Tables 4 and 5). We used the NEWS cut-points $>7$ or $>8$ which were the optimum cut-points. We found that NEWS $>8$ had the highest LR of 2.848 followed by NEWS $>7$ which had a LR of 2.180 which was the same as Corfield et al. ${ }^{16}$ Therefore, we suggest that patients with NEWS between 5 and 8 be transferred from the triage zone to the urgency zone and patients with NEWS $>8$ be transferred to the emergency zone immediately.

On the other hand, we preferred to use NEWS $\leq 4$ as the cut-point, indicating that the patient would probably have less diagnosis of sepsis, woud have low mortality, and could wait in the triage zone.

Our study has some limitations. It was retrospective in nature and conducted in a single center, an academic tertiary medical center. This could possibly restrict the generalizability of our results, due to limitations of health care in smaller hospitals. In addition, patients with multiple co-morbidities in our center, especially patients with advanced cancer or post-chemotherapy or both, comprised more than one-quarter of the patients in this study. These limitations may be solved by conducting a multi-center prospective trial in the future.

\section{Conclusion}

In summary, qSOFA is the most accurate scoring system for diagnosis sepsis which was consistent with previous study. However, qSOFA had the lowest sensitivity, so is not appropriate in a triage situation. Therefore, we decided to use NEWS as the triage tool because of its better sensitivity and acceptable specificity, as we need to triage almost all possible cases.

\section{Acknowledgement}

The authors thank Panita Worapratya for initiates' research, Kingkarn Waiyanak for article searches and retrieval and Glenn K. Shingledecker for his help in editing the manuscript. We also thank Alan Frederick Geater, Nannapat Pruphetkaew, and Walailuk Jitpiboon for the statistical assistance in the Epidemiology Unit, Faculty of Medicine, Prince of Songkla University.

\section{Conflict of interest}

The authors declares that he has no conflict of interest in this study.

\section{References}

1. Singer M, Deutschman CS, Seymour CW, Shankar-Hari M, Annane D, Bauer M, et al. The third international consensus definitions for sepsis and septic shock (Sepsis-3). JAMA 2016;315:801-10.

2. Seymour CW, Liu V, Iwashyna TJ, Frank MB, Thomas DR, Andre S, et al. Assessment of clinical criteria for sepsis. JAMA 2016;315:762-74

3. Haydar S, Spanier M, Weems P, Wood S, Strout T. Comparison of qSOFa and SIRS criteria as a screening mechanism for emergency department sepsis. Am J Emerg Med 2017;35: 1730-3.

4. Song JU, Sin CK, Park HK, Shim SR, Lee J. Performance of the quick Sequential (sepsis-related) Organ Failure Assessment score as a prognostic tool in infected patients outside the intensive care unit; a systematic review and meta-analysis. Crit Care 2018;22:28.

5. Maitra S, Som A, Bhattacharjee S. Accuracy of quick sequential organ failure assessment (qSOFA) score and systemic inflammatory response syndrome (SIRS) criteria for predicting mortality in hospitalized patients with suspected infection: a meta-analysis of observational studies. Clin Microbiol Infect 2018;24:1123-9. 
6. Finfer S, Bellomo R, Lipman J, French C, Dobb G, Myburgh J. Adult-population incidence of severe sepsis in Australian and New Zealand intensive care units. Intensive Care Med 2004; 30:589-96.

7. Martin GS, Mannino DM, Eaton S, Moss M. The epidemiology of sepsis in the United States from 1979 through 2000. N Eng J Med 2003;348:1546-54.

8. Vincent JL, Opal SM, Marshall JC, Tracey KJ. Sepsis definitions: time for change. Lancet 2013; 381:774-5.

9. Bozza FA, Carnevale R, Japiassú AM, Castro-Faria-Neto HC, Angus DC, Salluh Jl. Early fluid resuscitation in sepsis: evidence and perspectives. Shock 2010;34(Suppl 1):40-3.

10. Westphal GA, Koenig Á, CaldeiraFilho M, Feijó J, de Oliveira LT, Nunes F, et al. Reduce mortality after the implementation of a protocol for early detection of sepsis. J Crit Care 2011;26: 76-81.

11. Liu VX, Fieling-Singh V, Greene JD, Baker JM, Iwashyna TJ, Bhattacharya, et al. The timing of early antibiotics and hospital mortality in sepsis. Am J RespirCrit Care Med 2017;196:85663.

12. Raith EP, Udy AA, Bailey M, McGloughlin S, Maclsaac C, Bellomo $\mathrm{R}$, et al. Prognostic accuracy of the SOFA score, SIRS criteria, and qSOFA score for in-hospital mortality among adults with suspected infection admitted to the intensive care unit. JAMA 2017;317:290-300.

13. Askim $\AA$, Moser F, Gustad LT, Stene H, Gundersen M, Åsvold OB,et al. Poor performance of quick-SOFA (qSOFA) score in predicting severe sepsis and mortality - a prospective study of patients admitted with infection to the emergency department. Scand J Trauma Resusc Emerg Med 2017;25:56.

14. McLymont N, Glover GW. Scoring systems for the characterization of sepsis and associated outcomes. Ann Transl Med 2016;4:527.

15. Matthew MC, Ashley S, Xuan H, Sarah S, Natasha P, Michael $\mathrm{DH}$, et al. Quick sepsis-related organ failure assessment, systemic inflammatory response syndrome, and early warning scores for detecting clinical deterioration in infected patients outside the Intensive Care Unit. Am J Respir Crit Care Med 2017;195:906-11.

16. Corfield AR, Lees F, Zealley I, Houston G, Dickie S, Ward K, et al. Utility of a single early warning score in patients with sepsis in the emergency department. Emerg Med J 2014;31: $482-7$. 